\title{
Microscopic time-dependent analysis of neutrons transfers at low-energy nuclear reactions with spherical and deformed nuclei
}

\author{
Viacheslav Samarin \\ Joint Institute for Nuclear Research, 141980, Dubna, Moscow region, Russia
}

\begin{abstract}
Time-dependent Schrödinger equation is numerically solved by difference method for external neutrons of nuclei ${ }^{6} \mathrm{He},{ }^{18} \mathrm{O},{ }^{48} \mathrm{Ca},{ }^{238} \mathrm{U}$ at their grazing collisions with energies in the vicinity of a Coulomb barrier. The spin-orbital interaction and Pauli's exclusion principle were taken into consideration during the solution.
\end{abstract}

\section{Introduction}

It is generally accepted that the probability of neutron transfer reactions is highest during so-called grazing nuclear collisions. In this case, the distances between the surfaces of the atomic nuclei do not exceed the range of the action of nuclear forces (1-2 fm). The most probable transition is the one between the nuclei of the external, most weakly bound neutrons. In this study the spin-orbital interaction and Pauli's exclusion principle were taken into consideration. Time-dependent Schrödinger equation is numerically solved by difference method for external neutrons of spherical nuclei ${ }^{6} \mathrm{He}$, ${ }^{18} \mathrm{O},{ }^{48} \mathrm{Ca}$ and deformed nucleus ${ }^{238} \mathrm{U}$ at their grazing collisions with energies in the vicinity of Coulomb barrier. The visual computer animations of probability density evolution (http://nrv.jinr.ru/samarin/present/vsamarin2013.htm,

http://nrv.jinr.ru/samarin/present/vsamarin2013.ppt ) are calculated and probabilities of transfer of neutrons at reactions ${ }^{6} \mathrm{He}+{ }^{197} \mathrm{Au},{ }^{18} \mathrm{O}+{ }^{48} \mathrm{Ca},{ }^{40,48} \mathrm{Ca}+{ }^{238} \mathrm{U}$ are determined as function on minimum internuclear distances.

\section{Theory and numerical methods}

In the theoretical description of the neutron transfers upon heavy atomic nuclei collisions, a few semiclassical models are used [1-3]. They combine classical equations of atomic nuclei motion

$$
m_{1} \ddot{\vec{r}}_{1}=-\nabla_{r_{1}} V_{12}\left(\left|\vec{r}_{1}-\vec{r}_{2}\right|\right), m_{2} \ddot{\vec{r}}_{2}=-\nabla_{\vec{r}_{2}} V_{12}\left(\left|\vec{r}_{2}-\vec{r}_{1}\right|\right)
$$

that are justified by smallness of a de Broglie nuclear wave length and the quantum description of internal one-particle and collective degrees of freedom. Here $\vec{r}_{1}(t), \vec{r}_{2}(t)$ are nuclei centers with masses $m_{1}, m_{2}$ and $V_{12}(r)$ is the potential energy of nuclei interaction. Before contact between the surfaces of nuclei we may consider that neutron potential energy equal to sum

$$
V(\vec{r}, t)=V_{n}^{(1)}\left(\vec{r}-\vec{r}_{1}(t)\right)+V_{n}^{(2)}\left(\vec{r}-\vec{r}_{2}(t)\right)
$$


with spherically symmetric (for $\beta_{2}=0, \beta_{4}=0$ ) or axially symmetric with quadrupole and octupole dermations (for $\beta_{2} \neq 0, \beta_{4} \neq 0$ ) Woods-Saxon potential

$$
V\left(\vec{r}, \beta_{2}, \beta_{4}\right)=-V_{0}\left[1+\exp \left(\frac{r-R\left(\theta, \beta_{2}, \beta_{4}\right)}{a}\right)\right]^{-1} .
$$

The evolution of the components $\psi_{1}, \psi_{2}$ of the spinor wave function $\Psi(\vec{r}, t)$ of a neutron with mass $m$ during nuclei collisions is determined by time-dependent Schrödinger equation

$$
\frac{d}{d t}\left(\begin{array}{c}
\psi_{1} \\
\psi_{2}
\end{array}\right)=\left\{-\frac{\hbar^{2}}{2 m} \Delta+V(\vec{r}, t)-b \vec{\sigma}[(\nabla V) \hat{\vec{p}}]\right\}\left(\begin{array}{c}
\psi_{1} \\
\psi_{2}
\end{array}\right) .
$$

The numerical method of this equation solution [2] is similarly to split-operator fast Fourier transform (FFT) method without spin-orbital interaction [4]. The constant of the spin-orbital interaction $b$ can be presented in the form $b=0.022 R_{0}^{2} \kappa$, where $R_{0}=1 \mathrm{fm}$ and dimensionless constant $\kappa \sim 30$. At the present time, most of the deformed nuclei shell structure calculations based on Nilsson-Strutinsky method with expansion in harmonic oscillator wave functions (see, for example, [5,6]). We used alternative method offered in work [7] and based on a numerical solution of Schrödinger's equations at cylindrical coordinates with expansion in Bessel functions.

\section{Neutrons transfers at reactions with spherical nuclei}

We investigated collisions halo nucleus ${ }^{6} \mathrm{He}$ with spherical nucleus ${ }^{197} \mathrm{Au}$. The probability density $\rho$ for two external $1 \mathrm{p}_{3 / 2}$ shell neutrons of ${ }^{6} \mathrm{He}$ with averaging on total angular moment projection $\Omega$ to some axis

$$
\rho(\vec{r}, t)=\frac{1}{3} \sum_{\Omega=-3 / 2}^{3 / 2}\left(\left|\psi_{1}(\vec{r}, t)\right|^{2}+\left|\psi_{2}(\vec{r}, t)\right|^{2}\right)
$$

at frontal collision is shown in Fig. 1 $a$. The examples of evolution of the probability density are shown in report presentation: (http://nrv.jinr.ru/samarin/present/vsamarin2013.htm,

http://nrv.jinr.ru/samarin/present/vsamarin2013.ppt ). The formation of the stable structure of the probability density indicates that the neutrons preferably occupy two center state (Fig. 1a). After collision neutrons occupy several initially vacant levels above the Fermi level $\left(3 \mathrm{~d}_{5 / 2}, 4 \mathrm{~s}_{1 / 2}, 2 \mathrm{~g}_{7 / 2}, 3 \mathrm{~d}_{3 / 2}\right)$ in the ${ }^{197} \mathrm{Au}$ with an energy approximately equal to energy of external neutron in ${ }^{6} \mathrm{He}$ (about $-2 \mathrm{MeV}$ ).
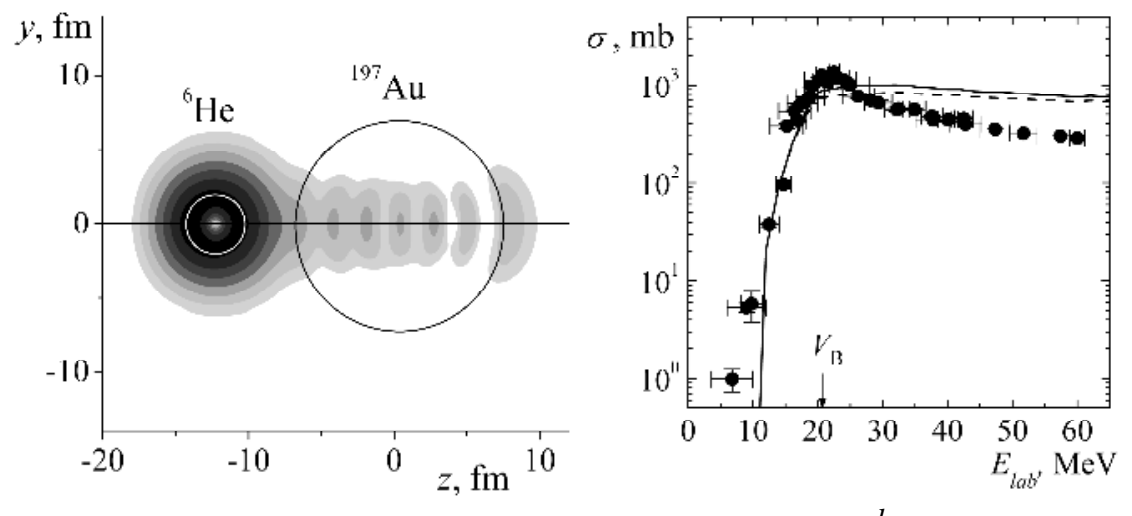

$a$

$b$

Figure 1. $a$ ) The probability density $\rho(x=0, y, z)$ of the external neutrons of ${ }^{6} \mathrm{He}$ nucleus with the initial state $1 \mathrm{p}_{3 / 2}$ during a collision with the ${ }^{197} \mathrm{Au}$ nucleus. $b$ ) Energy dependence of the cross section for the formation of the ${ }^{198} \mathrm{Au}$ isotope in the ${ }^{6} \mathrm{He}+{ }^{197} \mathrm{Au}$ reaction. Dots represent the experimental data from [8]; the dashed line calculations for the transfer of one neutron; the solid line - calculations for the transfer of one or two neutrons; $V_{\mathrm{B}}$ is the Coulomb barrier. 
The probability $p$ of neutron transfer during the collisions of nuclei without contact between their surfaces was determined in [3] by integrating the probability density over the volume and vicinity of the nucleus target after collision. The total cross section of the one or two neutrons transfer from shell, containing two neutrons before the collision is

$$
\sigma=2 \pi \int_{b_{0}}^{\infty} w(b) b d b,
$$

where $b_{0}$ is the minimum collision impact parameter $b$ corresponding to the grazing collision, when the surfaces of the nuclei approach the distance $a=0.7 \mathrm{fm}$, equal to the diffusivity of the surface region of nuclei. Function $w(b)$ is probability of transfer one or two neutrons [3]. A comparison of the experimental data on the cross section of neutron transfer during the ${ }^{6} \mathrm{He}+{ }^{197} \mathrm{Au}$ reaction and the calculation results demonstrates in Fig. $1 b$ satisfactory agreement between them at energies near the Coulomb barrier.

Pauli's exclusion principle limits transfers to occupied states for next studied reactions: ${ }^{18} \mathrm{O}+{ }^{48} \mathrm{Ca}$, ${ }^{48} \mathrm{Ca}+{ }^{238} \mathrm{U}$. This principle was taken into consideration by two approximations. On first simple approximation we except transfer to occupied levels in "frozen" shell structures of colliding nuclei, results are shown in Fig. $2 a$. On second approximation we used time dependent a few body wave function

$$
\Phi_{M}\left(\vec{r}_{1}, \ldots \vec{r}_{M}, t\right)=\frac{1}{\sqrt{M !}} \operatorname{det}\left[\begin{array}{ccc}
\Psi_{1}\left(\vec{r}_{1}, t\right) & \ldots & \Psi_{1}\left(\vec{r}_{N}, t\right) \\
\ldots & \ldots & \ldots \\
\Psi_{M}\left(\vec{r}_{1}, t\right) & \ldots & \Psi_{M}\left(\vec{r}_{M}, t\right)
\end{array}\right],
$$

for $M=2,3$. The probability $P$ of neutrons localization in vicinity of the nuclei was determined by corresponding integrating the $M$-body probability density

$$
P=\int\left|\Phi_{M}\left(\vec{r}_{1}, \ldots \vec{r}_{M}, t\right)\right|^{2} d V_{1} \ldots d V_{M}
$$

For neutron transfers at reaction ${ }^{18} \mathrm{O}+{ }^{48} \mathrm{Ca}$ two external neutrons $\left(1 \mathrm{~d}_{5 / 2}\right.$ from ${ }^{18} \mathrm{O}$ and $1 \mathrm{f}_{7 / 2}$ from $\left.{ }^{48} \mathrm{Ca}\right)$ with small total angular moment projection $\Omega=1 / 2,3 / 2$ to internuclear axis are took into account. For neutron transfers at reaction ${ }^{48} \mathrm{Ca}+{ }^{238} \mathrm{U}$ three external neutrons $\left(1 \mathrm{f}_{7 / 2}\right.$ in ${ }^{48} \mathrm{Ca}$ and $2 \mathrm{~g}_{9 / 2}, 1 \mathrm{i}_{11 / 2}$ in $\left.{ }^{238} \mathrm{U}\right)$ with $\Omega=1 / 2,3 / 2$ are took into account. Results for neutron transfer probability $p$ at reaction ${ }^{48} \mathrm{Ca}+{ }^{238} \mathrm{U}$ (Fig. $2 a, b$ ) demonstrate quality agreement between simple approximation with exception transfer to occupied states and a few body wave function approximation (7), (8).

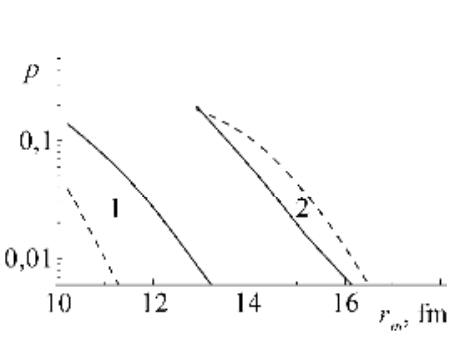

$a$

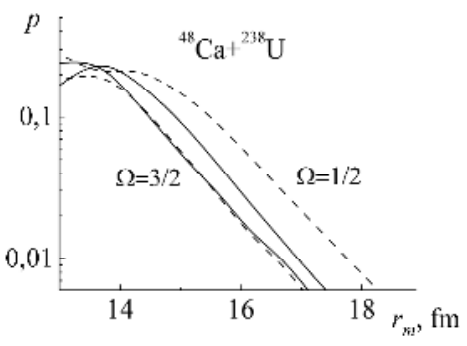

$b$

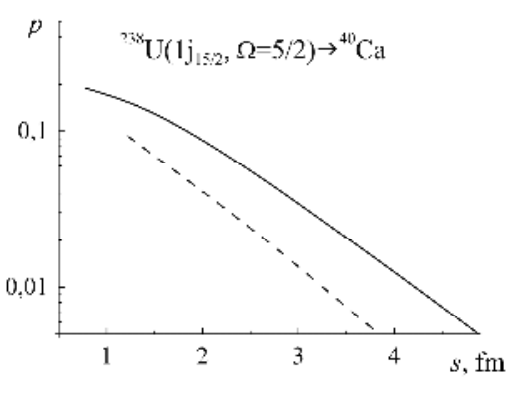

$c$

Figure 2. a) Probabilities of neutrons pick-up (solid curves) and stripping (dashed curves) for ${ }^{48} \mathrm{Ca}$ at frontal collision ${ }^{48} \mathrm{Ca}+{ }^{18} \mathrm{O}$ (curve 1) and ${ }^{48} \mathrm{Ca}+{ }^{238} \mathrm{U}$ (curve 2) as function on minimum value of internuclear distance $r_{m}$ in approximation with exception transfer to occupied states. $b$ ) Probabilities of neutrons pick-up (solid curves) and stripping (dashed curves) for ${ }^{48} \mathrm{Ca}$ at frontal collision ${ }^{48} \mathrm{Ca}+{ }^{238} \mathrm{U}$ as function $r_{m}$ in approximation (7), (8) with $M=3$ for small total angular moment projection $\Omega=1 / 2,3 / 2$.c) Probabilities of neutron stripping at reaction ${ }^{48} \mathrm{Ca}+{ }^{238} \mathrm{U}$ as a function of on minimum distance $s$ between nuclear surfaces. Angles between symmetry axes of deformed nucleus ${ }^{238} \mathrm{U}$ and initial velocity of ${ }^{40} \mathrm{Ca}$ nuclei equal to $45^{\circ}$ (solid line) and $90^{\circ}$ (dashed line). 
At reactions ${ }^{6} \mathrm{He}+{ }^{197} \mathrm{Au},{ }^{18} \mathrm{O}+{ }^{48} \mathrm{Ca}$ neutrons are predominantly transferred along internuclear axis from a smaller nucleus to the greater nucleus. At reaction ${ }^{48} \mathrm{Ca}+{ }^{238} \mathrm{U}$ probabilities of neutrons stripping and pick-up are commensurable.

\section{Neutrons transfers at reactions with deformed nucleus}

Some upper energy levels for neutron states with module of total angular moment projection to symmetry axis $\Omega$ at axially deformed nucleus ${ }^{238} \mathrm{U}$ with deformation parameters $\beta_{2}=0.215$ and $\beta_{4}=0.095$ are shown on Fig. $3 a$. Probability densities of external neutron of ${ }^{238} \mathrm{U}$ for initial state $1 \mathrm{j}_{15 / 2}$ with $\Omega=5 / 2$ (upper occupied level, Fig. $3 a$ ) during frontal collision with the nucleus ${ }^{40} \mathrm{Ca}$ are shown on Fig. $3 b$. Neutron wave function penetrates into neighbor well along internuclear axis. The probabilities of neutron pick-up at reaction ${ }^{40} \mathrm{Ca}+{ }^{238} \mathrm{U}$ are shown in Fig. $2 c$ as a function of minimum distance $s$ between nuclear surfaces for two of possible orientations of deformed nucleus ${ }^{238} \mathrm{U}$.

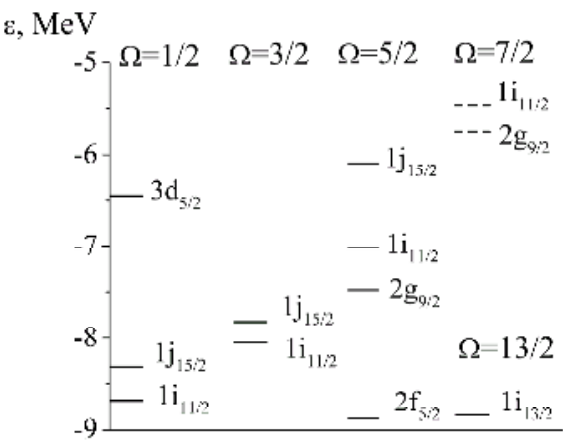

$a$

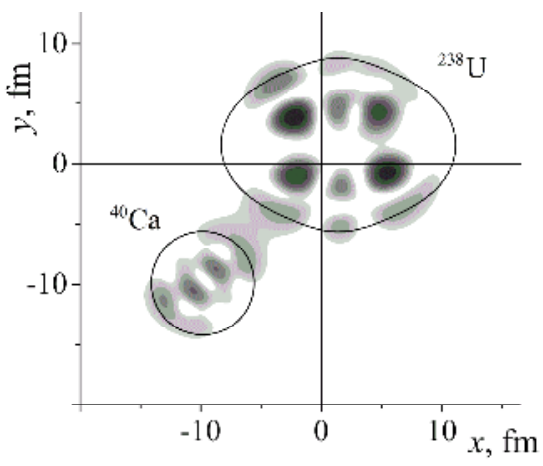

$b$

Figure 3. a) Some upper energy levels for neutron states with module of total angular moment projection to symmetry axis $\Omega$ at nucleus ${ }^{238} \mathrm{U}$ with deformation parameters $\beta_{2}=0.215$ and $\beta_{4}=0.095$, dashed lines correspond unoccupied levels. $b$ ) Probability density $\rho(x, y, z=0)$ of the external neutron of ${ }^{238} \mathrm{U}$ for initial state $1 j_{15 / 2}$ with $\Omega=5 / 2$ during frontal collision with the ${ }^{40} \mathrm{Ca}$ at energy in the center of mass system $E=192 \mathrm{MeV}$. Angle between symmetry axes of deformed nucleus ${ }^{238} \mathrm{U}$ and initial velocity of ${ }^{40} \mathrm{Ca}$ nuclei equal to $45^{\circ}$.

This work was supported in part by the Russian Foundation for Basic Research (RFBR) through Grant No 12-02-01325-a.

\section{References}

1. A. Winther, Nucl. Phys. A 572. 191 (1994)

2. V. Samarin, K. Samarin, Bull. Russ. Acad. Sci. Phys. 74. 567 (2010)

3. V. Samarin, K. Samarin, Bull. Russ. Acad. Sci. Phys. 76. 450 (2012)

4. M. Riley, B. Ritchie, Phys. Rev. A 59, 3544 (1999)

5. K. Langanke, J. Maruhn, S. Koonin, Computational Nuclear Physics 1. Nuclear Structure. (Springer-Verlag, Germany, 1991)

6. Nuclear Reaction Video (Database of Low-Energy Nuclear Reactions, FLNR, JINR, Dubna), http://nrv.jinr.ru/nrv/

7. V. Samarin, Phys. of Atom. Nucl. 73. 1416 (2010)

8. A. Kulko, N. Demekhina, R. Kalpakchieva, N. Kolesnikov, V. Lukashik, Yu. Penionzhkevich, D. Rassadov, N. Skobelev., J. Phys. G 34. 2297 (2007) 